\title{
Wearable Technology: Signal Recovery of Electrocardiogram From Short Spaced Leads in the Far-Field Using Discrete Wavelet Transform Based Techniques
}

\author{
Niamh McCallan ${ }^{1}$, Dewar Finlay ${ }^{1}$, Pardis Biglarbeigi ${ }^{1}$, Gilberto Perpiñan ${ }^{2}$, Michael Jennings ${ }^{1}$, Kok \\ Yew $\mathrm{Ng}^{1}$, James McLaughlin ${ }^{1}$, Omar Escalona ${ }^{1}$ \\ ${ }^{1}$ NIBEC, Ulster University, Newtownabbey, United Kingdom \\ ${ }^{2}$ Universidad Antonio Nariño, Cartagena, Colombia
}

\begin{abstract}
Bipolar ECG leads recorded from closely spaced electrodes are challenging in any context. When they are positioned distally with respect to the source field (far-field), the recovery of clinically useful signal content represents an even greater challenge. Due to the increased interest in ambulatory wellness devices, particularly wrist-worn devices, there is a renewed interest in recovering ECG signals from distally located bipolar leads.

In this study 10 bipolar leads were simultaneously recorded at various locations along the left arm. At the same time, a conventional proximal reading on the chest using Lead I was also recorded and stored. This process was repeated for 11 healthy subjects. ECGs were recorded for a period of approximately 6 minutes for each subject and sampled at a frequency of $2048 \mathrm{~Hz}$. Wavelet-based filtering using Daubechies 4 wavelet decomposition and soft threshold was applied to each lead. QRS detection performance was assessed against Lead I for each subject.

This investigation found that a lead positioned transversally (using BIS gelled electrodes) on the upper arm provided the best accuracy against the benchmark QRS detection $(S E N=0.998, P P V=0.984)$. The most distally positioned bipolar lead using dry electrodes faired least favourable $(S E N=0.272, P P V=0.202)$.
\end{abstract}

\section{Introduction}

In recent years there has been a move from stationary to portable ECG devices i.e. wearable ECGs. With portability comes increased mobility and movement, in terms of accuracy this creates more unwanted noise [1]. To maximise ECG signal quality, electrodes are positioned either on standardised locations along the chest wall or on both arms. This however becomes problematic when considering long term monitoring for multiple reasons;

1. Standard daily activity which restricts free motion.
2. Electrode adhesion, which causes irritation to the skin after only a short time attached [2].

Therefore, electrodes are ideally positioned in a more convenient and comfortable location e.g. wrist-worn. This however results in an almost undetectable ECG signal due to electrode spacing and smaller cardiac signal in distant locations caused by muscle artefact [3]. Much research has gone into the exploration and development of wearable ECG monitors, with portable and wireless devices being one of the most in-demand and desirable bio-medical measurement technologies [4]. As electronic equipment is becoming smaller, the size of monitoring devices can also be reduced [5]. Furthermore, the necessity to create functional wearable ECG devices with a reduced interelectrode distance has increased [6]. However, reduction of the inter-electrode distance essentially causes the signal strength to reduce [7]. Consequently, the main challenge of this investigation is the ability to develop a filtering technique to effectively denoise the poor signals produced from different regions along the arm. Wearable Health Devices (WHDs), mainly wrist-worn monitors, have become a phenomenon [5]. Currently, technology exists for measuring heart rate in the form of optical photoplethysmogram (PPG). However, this technology cannot accurately detect heart rhythm [8]. Some emerging devices are providing ECG based functionality; however, these devices rely on the user touching the device on their wrist with their other hand in order to establish a bipolar lead. A bipolar lead measured from a single device on one wrist still remains elusive [9]. This study aims to provide a comprehensive method for the recovery of ECG information located in far field sites.

\section{Methods}

In this study, several bipolar leads at various positions along the left arm have been recorded to assess their prevalence over the background noise. The background noise 
has been removed using noise filtering techniques in order to detect the QRS complex of each lead along the left arm.

\subsection{Data Acquisition}

In this study, data was gathered from Craigavon Area Hospital (CAH), Northern Ireland in association with WASTCArD project. Arm ECGs of 11 subjects were recorded in a resting position for a period of 5 to 6 minutes, using 10 bipolar leads. Recordings were completed on various days utilizing the same equipment and room conditions. A combination of dry and special pre-gelled BIS Quatro $^{\mathrm{TM}}$ electrodes were positioned axially and transversally along the left arm as depicted in Table 1 and Figure 1 [10].

\begin{tabular}{l|l|l}
\hline Leads & Channels & Location \\
\hline \multicolumn{3}{c}{ DRY EleCtRODE LEADS } \\
\hline Lead 1 & Ch13-Ch14 & Upper Arm \\
Lead 3 & Ch11-Ch12 & Wrist \\
Ch13-Ch12 & Wrist-Upper Arm \\
\hline \multicolumn{3}{c}{ TrANSVERSAL BIS GEL ElECTRODE LEADS } \\
\hline Lead 4 & Ch10-Ch8 & Upper Arm \\
Lead 5 & Ch7-Ch5 & Lower Arm \\
Lead 6 & Ch4-Ch3 & Wrist \\
\hline \multicolumn{3}{c}{ AXIAL BIS GEL ELECTRODE LEADS } \\
\hline Lead 7 & Ch10-Ch6 & Upper Arm-Forearm \\
Lead 8 & Ch7-Ch2 & Forearm-Wrist \\
Lead 9 & Ch10-Ch2 & Upper Arm-Wrist \\
Lead 10 & Ch1-Ch3 & Lower Arm - Right Arm \\
\hline \multicolumn{3}{c}{ ReFERENCE LEAD } \\
\hline Lead I & Ch16-Ch17 & Left Arm-Right Arm \\
\hline
\end{tabular}

Table 1. Lead Numbers and Anatomical Locations of Studied Left-Arm ECG Bipolar Leads.

MATLAB R2018b (The Mathworks Ins., Natick, MA, USA) was used as the signal processing tool for the ECG WASTCArD data provided [11]. All subjects signed a consent form in agreement with the study. Ethical approval was obtained from HSC REC B (Health and Social Care, Research Ethical Committee, Reference: 16/NI/0158), and IRAS (Integrated Research Application System, Registered Project ID: 203125, Dated: 21/September/2016).

\subsection{Pre-processing}

Firstly, each lead was extracted from the acquisition channels as described in Table 1. Then the DC component of each lead was removed by subtracting the mean value of the ECG signal using Equation 1.

$$
E C G_{D C R}=E C G_{\text {input }}-\operatorname{mean}\left(E C G_{\text {input }}\right)
$$

where $E C G_{D C R}$ represents the output signal with the DC

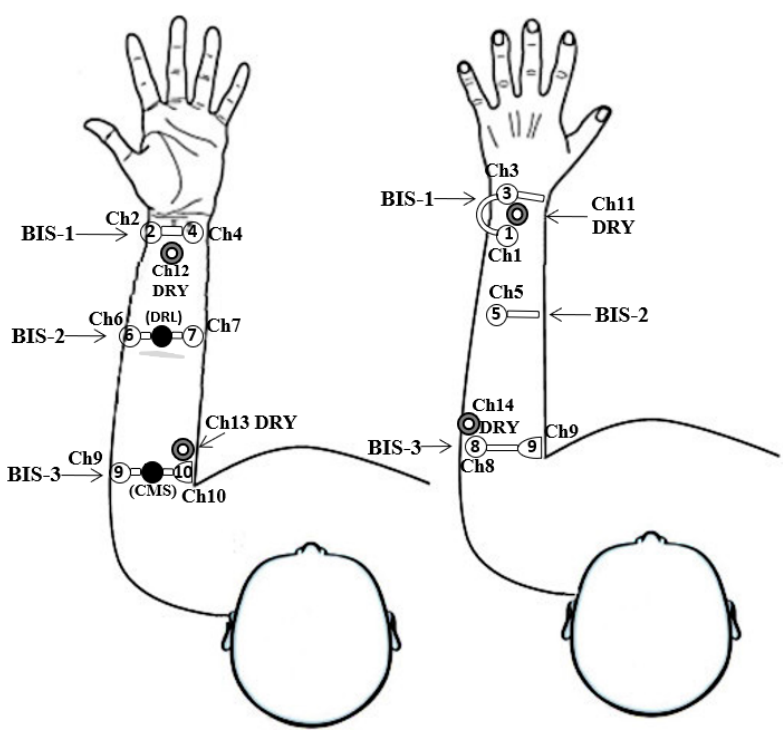

Figure 1. Anatomical locations of leads along the left arm using the BIS-QuatroTM sensor strips and dry electrodes.

component removed and $E C G_{\text {input }}$ represents the original ECG signal.

A notch filter set to $50 \mathrm{~Hz}$ was used to remove the power-line interference. This is most commonly experienced due to the simple problem of disconnected electrodes and therefore requires immediate re-connection. Otherwise, this signal interference would result in poor quality tracing. Furthermore, a second order high pass Butterworth filter at $0.5 \mathrm{~Hz}$ was used to limit noise due to respiratory artefact. Also, a second order low pass Butterworth filter at $40 \mathrm{~Hz}$ was used to limit the bandwidth of the signal and attenuate out band noise and the power-line interference [2].

\subsection{DWT-based denoising algorithm}

Discrete Wavelet Transforms (DWT) have been demonstrated as a powerful tool for ECG signal analysis [12]. It not only localizes the information of signals in the timefrequency domain, but is also capable of trading one type of resolution for the other, which makes it especially suitable for the analysis of non-stationary signals such as ECG signals [13].

For this reason, wavelet-based methods of denoising are becoming more advantageous signal analysis techniques offering flexibility and adaptability to overcome limitations associated with standard filtering techniques [14]. Furthermore, this method can be useful when resolving issues such as power-line interference and internal muscle movement.

For this study, ECG signals were denoised using a $4^{\text {th }}$ 
level wavelet decomposition with db4 Daubechies wavelet as the mother wavelet. The $4^{t h}$-order wavelet was selected over the $6^{\text {th }}$-order wavelet due to its similarity with the QRS complex [15]. The signal is broken down into 4 levels of approximated coefficients and detailed coefficients, as presented in Figure 2.

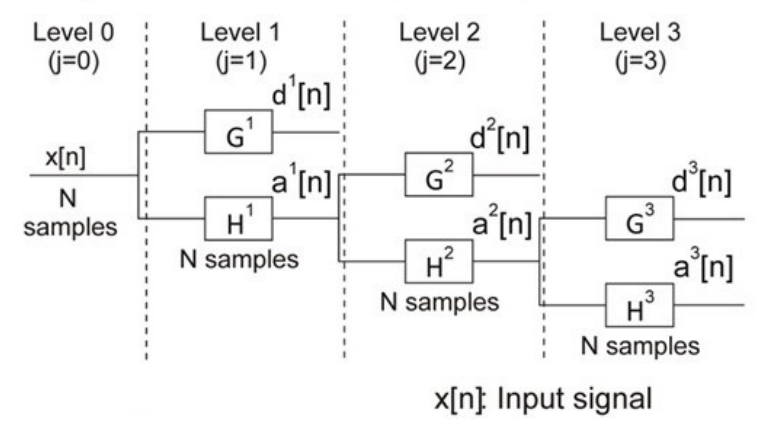

Figure 2. An example of a $3^{\text {rd }}$ level Wavelet Decomposition [16]

The approximate coefficients $(\mathrm{H})$ represent the low frequency components obtained using low pass filters and the detailed coefficients $(G)$ represent the high frequency components obtained using high pass filters. From these coefficients, a soft threshold was applied to modify the signal where every coefficient higher than the threshold value is decreased by the size of the threshold. The remaining coefficients that are lower than the threshold are then set to zero. The threshold $\lambda$ can be determined using the following equation:

$$
\lambda=\sigma \log N
$$

where the standard deviation is represented by $\sigma$ and the length of the signal is represented by $N$.

\subsection{Performance Assessment}

Given the absence of a gold standard waveform at the sites. Where the bipolar leads were recorded, it is not possible to compare the recorded waveform to an actual signal. Therefore, performance assessment was made based on the ability to accurately detect QRS complexes on the ECG signals recorded from each of the distally recorded ECG lead. This approach was adopted as any ambulatory system is likely to rely on accurate QRS detection, and subsequent R-wave location. To establish basic measurements (rate and rhythm). In the conduct of this research, a computer algorithm was used to detect QRS complexes on each of the recorded signals. This algorithm is based on the Pan and Tompkins approach [17], which has been optimised for wearable/ambulatory applications. Annotations obtained using the algorithm from the proximally recorded Lead I were used as the reference annotations. Annotations from each lead, again obtained using the computer algorithm, were compared to the reference annotations from Lead I. Comparison of reference and test annotations were based on the ANSI/AAMI-standard beat-by-beat annotation comparator reference implementation that is available in the PhysioNet WaveForm DataBase (WFDB) Toolbox [1]. The performance evaluation was configured considering all annotations, from the beginning of each record. Reference and test annotations were considered equivalent when they fell within the reference implementations default $150 \mathrm{~ms}$ window. Performance was expressed as percentage of sensitivity, the ratio of correct detections to the actual number of beats, and positive predictive value (PPV), the ratio of correct detections to the total number of beats detected:

$$
\begin{aligned}
S e & =\frac{T P}{T P+F N} \cdot 100 \% \\
P P V & =\frac{T P}{T P+F P} \cdot 100 \%
\end{aligned}
$$

where True Positives (TP) represents the peaks that have been correctly identified, False Positive (FP) is equal to a point that has been incorrectly identified as a peak, and False Positive $(\mathrm{FN})$ represents any peak that has not been identified [10].

\section{Results and Discussion}

Signal noise appears to be more evident when the dry electrodes are used. This is likely due to the interaction between the subjects' skin and the electrode, which in turn creates a rubbing effect [2]. Lead 2, a dry electrode located on the wrist, performed with a median sensitivity of only 0.292 and PPV of 0.191 as presented in Table 2.

\begin{tabular}{l|r|r|r|r}
\hline LEADS & SENS(MED) & PPV(MED) & SENS(MEAN) & PPV(MEAN) \\
\hline $\mathbf{1}$ & $\mathbf{0 . 7 2 6}$ & $\mathbf{0 . 6 6 7}$ & $\mathbf{0 . 5 3 7}$ & $\mathbf{0 . 5 4 4}$ \\
\hline $\mathbf{2}$ & $\mathbf{0 . 2 9 2}$ & $\mathbf{0 . 1 9 1}$ & $\mathbf{0 . 2 7 2}$ & $\mathbf{0 . 2 0 2}$ \\
\hline 3 & 0.814 & 0.493 & 0.627 & 0.535 \\
\hline $\mathbf{4}$ & $\mathbf{1}$ & $\mathbf{0 . 9 9 4}$ & $\mathbf{0 . 9 9 8}$ & $\mathbf{0 . 9 8 4}$ \\
\hline 5 & 0.494 & 0.234 & 0.382 & 0.217 \\
\hline $\mathbf{6}$ & $\mathbf{0 . 4 1 1}$ & $\mathbf{0 . 2 1 9}$ & $\mathbf{0 . 3 7 3}$ & $\mathbf{0 . 2 1 9}$ \\
\hline 7 & 0.983 & 0.975 & 0.845 & 0.787 \\
\hline 8 & 0.452 & 0.2 & 0.315 & 0.174 \\
\hline 9 & 0.93 & 0.683 & 0.828 & 0.646 \\
\hline 10 & 0.88 & 0.227 & 0.388 & 0.218 \\
\hline I & 1 & 1 & 1 & 1 \\
\hline
\end{tabular}

Table 2. Sensitivity and PPV of the Mean and Median for all the subjects.

As for Lead 6, a conductive gel electrode that is also located on the wrist, the median sensitivity achieved was sufficiently larger than that of Lead 2 at 0.411 . Overall, the 
best performing lead was Lead 4 located on the upper arm, with a median sensitivity of 1 and PPV of 0.994 , which was used with a conducting gel electrode. Lead 1, also located on the upper arm, achieved weaker results as seen in Table 2. This provides further evidence that the introduction of gel electrodes seems to alleviate the issue of the rubbing effect created by dry electrode and consequently giving better results for the detection of the QRS complex. Better QRS detection may also be affected by electrode spacing. In the results gathered, the shortest spaced leads i.e Lead 2 and Lead 6 has the lowest sensitivity and PPV values, whereas leads 1 and 4, with greater electrode spacing, performed the best.

\section{Conclusion}

The use of DWT for ECG information recovery in far field sites were investigated in this study. These findings determined that a reasonable level of QRS detection accuracy can be achieved from electrode positioned on the upper arm with greater electrode spacing. Overall, optimum results were obtained from conductive gel electrodes. Whilst the quality of the ECG signals may not be adequate for comprehensive clinical interpretation this level of accurate QRS detection may still be useful in applications such as heart rate variability analysis. However, due to the fact that the study was carried out with only 11 subjects, a more substantial database needs to be collected for further investigation.

\section{Acknowledgments}

This ongoing research is supported by funding by the Connected Health Innovation Centre (CHIC) and the European Union (EU): H2020-MSCA-RISE Programme (WASTCArD Project, Grant \#645759).

\section{References}

[1] Silva I, Moody GB. An open-source toolbox for analysing and processing physionet databases in matlab and octave. Journal of Open Research Software 2014;2(1).

[2] Escalona O, Lynn W, Perpiñan G, McFrederick L, McEneaney D. Data-driven ecg denoising techniques for characterising bipolar lead sets along the left arm in wearable long-term heart rhythm monitoring. Electronics 2017; 6(4):84

[3] Massot B, Noury N, Gehin C, McAdams E. On designing an ubiquitous sensor network for health monitoring. In 2013 IEEE 15th International Conference on e-Health Networking, Applications and Services (Healthcom 2013). IEEE, 2013; 310-314.

[4] Mukhopadhyay SC. Wearable sensors for human activity monitoring: A review. IEEE Sensors Journal 2014; 15(3):1321-1330.
[5] Dias D, Paulo Silva Cunha J. Wearable health devicesvital sign monitoring, systems and technologies. Sensors 2018; 18(8):2414.

[6] Yang Z, Zhou Q, Lei L, Zheng K, Xiang W. An iot-cloud based wearable ecg monitoring system for smart healthcare. Journal of Medical Systems 12 2016;40:286.

[7] Puurtinen M, Viik J, Hyttinen J. Best electrode locations for a small bipolar ecg device: signal strength analysis of clinical data. Annals of Biomedical Engineering 2009; 37(2):331-336.

[8] Georgiou K, Larentzakis AV, Khamis NN, Alsuhaibani GI, Alaska YA, Giallafos EJ. Can wearable devices accurately measure heart rate variability? a systematic review. Folia Medica 2018;60(1):7-20.

[9] Ip JE. Wearable devices for cardiac rhythm diagnosis and management. Jama 2019;321(4):337-338.

[10] Goodfellow J, Escalona OJ, Kodoth V, Manoharan G, Bosnjak A. Denoising and automated r-peak detection in the ecg using discrete wavelet transform. In 2016 Computing in Cardiology Conference (CinC). IEEE, 2016; 1045-1048.

[11] Steinhaus S. Comparison of mathematical programs for data analysis, 2008.

[12] Addison PS. Wavelet transforms and the ecg: a review. Physiological Measurement 2005;26(5):R155.

[13] He H, Wang Z, Tan Y. Noise reduction of ecg signals through genetic optimized wavelet threshold filtering. In 2015 IEEE International Conference on Computational Intelligence and Virtual Environments for Measurement Systems and Applications (CIVEMSA). IEEE, 2015; 1-6.

[14] Singh BN, Tiwari AK. Optimal selection of wavelet basis function applied to ecg signal denoising. Digital Signal Processing 2006;16(3):275-287.

[15] Patil PB, Chavan MS. A wavelet based method for denoising of biomedical signal. In International Conference on Pattern Recognition, Informatics and Medical Engineering (PRIME-2012). IEEE, 2012; 278-283.

[16] Quotb A, Bornat Y, Renaud S. Wavelet transform for realtime detection of action potentials in neural signals. Frontiers in Neuroengineering 2011;4:7.

[17] Pan J, Tompkins WJ. A real-time qrs detection algorithm. IEEE Trans Biomed Eng 1985;32(3):230-236.

Address for correspondence:

Niamh McCallan

NIBEC, Ulster University,

Newtownabbey,

United Kingdom,

BT37 0QB

mccallan-n2@ulster.ac.uk 